\title{
Neuropéptidos en el encéfalo humano
}

\author{
Neuropeptides in the human brain

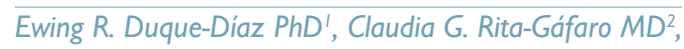 \\ Marcela Bermúdez-Echeverry PhD
}

\begin{abstract}
Resumen: Los estudios en el encéfalo humano se han realizado con el fin de responder a varias preguntas de carácter científico relacionados con la neuroanatomía, neurofisiología, neurofarmacología, la neurología y la conducta. El encéfalo es el órgano en el que se encuentra la regulación de los reflejos y los mecanismos inconscientes como la transmisión del dolor, los cardiovasculares, los respiratorios, entre otros. Estos mecanismos están mediados por sustancias químicas tales como los neuropéptidos, que son cadenas cortas de aminoácidos que se han encontrado ampliamente distribuidos en el sistema nervioso central y periférico, además de ejercer acciones fisiológicas actuando como neurotransmisores, neuromoduladores (acciones paracrinas y autocrinas) y neurohormonas. En los últimos treinta años se ha incrementado el conocimiento sobre la distribución y función de los neuropéptidos en el sistema nervioso central de mamíferos (ratas, gatos, perros, alpacas, primates y humanos). Así, el objetivo de esta revisión se dirige a describir los datos más relevantes disponibles sobre los neuropéptidos en el encéfalo humano. Para ello se revisan aspectos importantes de los neuropéptidos en el encéfalo humano como: a) La distribución, b) Las relaciones anatómicas, c) Las funciones fisiológicas, d) La coexistencia, y e) Las investigaciones futuras a realizar.
\end{abstract}

Palabras clave: Neuropéptidos, encéfalo, tronco encefálico, núcleos encefálicos.

Abstract: The human brain has been used in laboratory as an experimental model in order to answer several scientific questions related to neuroanatomy, neurophysiology, neuropharmacology, neurology and behavior. The brain is the organ in which the regulation of reflexes and "unconscious» mechanisms (transmission of pain, cardiovascular, respiratory, etc.) are carried out. These mechanisms are

\footnotetext{
' Biólogo, PhD en Neurociencias. Docente investigador, Coordinador del Grupo de Neurociencias UDES. Escuela de Medicina, Universidad de Santander (UDES). Bucaramanga, Colombia. Correspondencia: Calle 70 \# 55-2 10. Campus Lago del Cacique. Teléfono: 5776516500 ext. 1382. Fax: 577 6516492. Correo electrónico: coord.neurociencias@udes.edu.co

${ }^{2}$ Médico, rural en ESE Hospital el Carmen, San Vicente de Chucurí. Joven Investigador, Grupo de Neurociencias UDES. Escuela de Medicina, Universidad de Santander (UDES). Bucaramanga, Colombia.

${ }^{3}$ Médico, MSc en Psicobiología, PhD en Fisiología Humana. Docente investigador, Grupo de Neurociencias UDES. Escuela de Medicina, Universidad de Santander (UDES). Bucaramanga, Colombia.

Conflicto de intereses: los autores declaran que no tienen conflicto de intereses Medicina \& Laboratorio 2015; 21 : 349-362

Módulo 2 (Endrocrinología), número 13. Editora Médica Colombiana S.A. $2015^{\odot}$

Recibido el 09 de julio de 2015; aceptado el 03 de agosto de 2015
} 
mediated by chemicals such as neuropeptides, which are shorter chains of amino acids that have been showed widely distributed in the central and peripheral nervous system and to exert physiological actions acting as neurotransmitters, neuromodulators (paracrine action and autocrine) and neurohormones. In the last thirty years, it has increased the knowledge on the distribution and function of neuropeptides in the central nervous system of mammals (rats, cats, dogs, alpaca, primates, and humans). Thus, the aim of this paper is to describe the most relevant information available about neuropeptides in the human brain. To do so will raise issues about neuropeptides in the human brain as: a) The distribution; b) The anatomical relationship; c) The physiological functions; d) The coexistence; and e) The related future research to make.

Key words: Neuropeptides, brain, brain stem, brain nuclei.

Duque-Díaz ER, Rita-Gáfaro CG, Bermúdez-Echeverry M. Neuropéptidos en el encéfalo humano. Medicina \& Laboratorio 2015; 21 : 349-362.

L os neuropéptidos son cadenas cortas de aminoácido que se sintetizan en el sistema nervioso. Estas sustancias actúan como neurotransmisores, neuromoduladores y neurohormonas; están envueltas en acciones tales como la ingesta de agua y alimentos, la termorregulación, la analgesia, la nocicepción, la ansiedad, el control neuroendocrino, la regulación gastrointestinal, la regulación del sistema inmune, la inflamación, la regulación del páncreas y los riñones, el control en la liberación de los neurotransmisores clásicos, así como también en mecanismos sexuales, respiratorios, cardiovasculares, visuales, neuroprotectores, de memoria y aprendizaje [I]. Además, se conoce que estas sustancias están ampliamente distribuidas en el sistema nervioso central de los mamíferos [2].

Hasta los años ochenta la distribución de los neuropéptidos en el sistema nervioso central no había sido estudiada a profundidad. A partir de entonces, al menos en el ser humano, este conocimiento, así como el referente a la función fisiológica de los neuropéptidos se incrementó notablemente; sin embargo, hoy en día aún hay muchos vacíos, especialmente sobre la distribución de estas sustancias neuroactivas en el sistema nervioso central [3]. De esta manera, esta revisión se centra en una consulta pormenorizada de la bibliografía científica referente a la distribución cartográfica de los neuropéptidos observados en el encéfalo humano, teniendo en cuenta técnicas como la inmunocitoquímica, la hibridación in situ, el radioinmunoensayo y la cromatografía, entre otros. Además, se incluye la experiencia de nuestro grupo de investigación en cuanto a la distribución de los neuropéptidos en el sistema nervioso central de los mamíferos.

\section{Distribución de neuropéptidos en el encéfalo humano}

Una de las grandes familias que agrupan a los neuropéptidos son los opiáceos, que se divide en tres precursores: a) los derivados de la proopiomelanocortina (POMC) como la $\alpha$-endorfina, la $\gamma$-endorfina, la $\beta$-endorfina $(|-3|)$, la $\beta$-endorfina $(\mid-27)$ y el péptido no opiáceo, la hormona adrenocorticotropina (ACTH); b) los derivados de la proencefalina como la metionina-encefalina-Arg-Phe ${ }^{7}$, la metionina-encefalina-Arg-Gly ${ }^{7}-$ Leu $^{8}$ (Met-8) y la 
leucina-encefalina y c) los derivados de la prodinorfina como la dinorfina A ( I-8), la dinorfina $B$ (rimorfina), la $\alpha$-neoendorfina y la $\beta$-neoendorfina. En los invertebrados también se produce un péptido que contiene una secuencia de aminoácidos similar a la metioninaencefalina-Arg ${ }^{6}-$ Phe $^{7}$ llamada FMRFamida [4], la cual está involucrada en diversas funciones del sistema nervioso central de los vertebrados [5-7]. Así, varios péptidos opiáceos han sido mapeados en el encéfalo humano: la encefalina [8,9], la leucina-encefalina $[10,1$ I ], la metionina 5-encefalina-Arg-Gly -Leu $^{8}$ [I2], la metionina-encefalina-Arg-Gly ${ }^{7}-$ Leu $^{8}$ (Met-8) [I 3], la $\alpha$-neoendorfina [3] y la $\beta$-endorfina $[14,15]$.

Mediante el uso de la técnica de radioinmunoensayos Rinne y colaboradores (I993) [ I 0, I I] demostraron la presencia de las encefalinas en el encéfalo humano, con base en la observación de receptores para la metionina-encefalina y leucina-encefalina en diferentes áreas del cerebro de individuos post mortem que tuvieron un envejecimiento normal o presentaron demencias vascular y tipo Alzheimer. En estos trabajos, los individuos con demencia presentaron un bajo número de receptores en regiones como el globo pálido, la amígdala, el hipocampo y la corteza temporal, lo que sugiere una relación directa con la unión de las encefalinas y la dopamina, que a su vez puede generar la pérdida de neuronas y la ruptura sináptica que genera un evento primario en la patología. En cuanto a los resultados en el encéfalo envejecido se encontró un bajo número de receptores para encefalinas en el globo pálido y el núcleo caudado, pero las uniones de estos receptores en vías dopaminérgicas (sustancia negra y putamen) se mantienen, lo que sugiere que el descenso en ciertas áreas y la preservación en otras tienen un efecto con poca significancia sobre el envejecimiento del sistema nervioso.

La presencia de las encefalinas en el encéfalo humano también se ha demostrado usando la técnica de inmunocitoquímica en encéfalos post mortem de individuos con demencia presenil y senil [8]. En este trabajo, Bouras y colaboradores (1984) demostraron la presencia de estructuras inmunorreactivas (fibras) en regiones como el núcleo accumbens, el núcleo caudado, el globo pálido, el núcleo septal, la sustancia innominada, el hipotálamo, la hipófisis, la sustancia negra, el núcleo interpeduncular, el locus coeruleus y otros núcleos del tronco del encéfalo. Esta amplia distribución es muy similar a la encontrada en estudios más recientes realizados en primates por nuestro grupo de investigación [1-3]; sin embargo, no se estableció una relación directa de esta distribución con la condición clínica del estudio anterior.

Utilizando la técnica de inmunocitoquímica Coveñas y colaboradores (2004) [13] también hallaron estructuras inmunorreactivas que contenían metionina-encefalina-Arg-Gly - Leu $^{8}$ (Met-8) en regiones del tronco del encéfalo humano, como el tracto del núcleo solitario, el núcleo hipogloso, el núcleo vestibular medial y espinal, el núcleo cuneato lateral, el núcleo vestibular, el núcleo praepusitus, el colículo inferior, la parte ventral de la oliva superior y en medio del puente y el mesencéfalo. Además, observaron una alta densidad de fibras marcadas con Met-8 en núcleos como el núcleo espinal del trigémino, la sustancia gris periacueductal, la formación reticular mesencefálica, pontina y bulbar, el núcleo solitario, el núcleo vestibular espinal, el núcleo olivar dorsal, el núcleo del rafe, la sustancia negra y el núcleo interpeduncular.

Por su parte, en un estudio inmunocitoquímico reciente realizado por Duque y colaboradores (20।3) [3] se demostró la presencia de una alta, moderada y baja densidad de estructuras 
inmunorecativas (somas y fibras) que contienen $\alpha$-neo-endorfina en el tronco del encéfalo humano. En este estudio se evidenció la presencia de este neuropéptido en áreas como la sustancia gris periacueductal (región medular), el núcleo espinal del trigémino (pars gelatinosa y magnocelular) (véase figura I), la parte caudal del núcleo solitario, la formación reticular, el núcleo cuneato lateral (véase figura 2), el núcleo gracilis, el núcleo motor del trigémino, el núcleo ambiguo, el colículo superior y el núcleo hipogloso, entre otros núcleos.
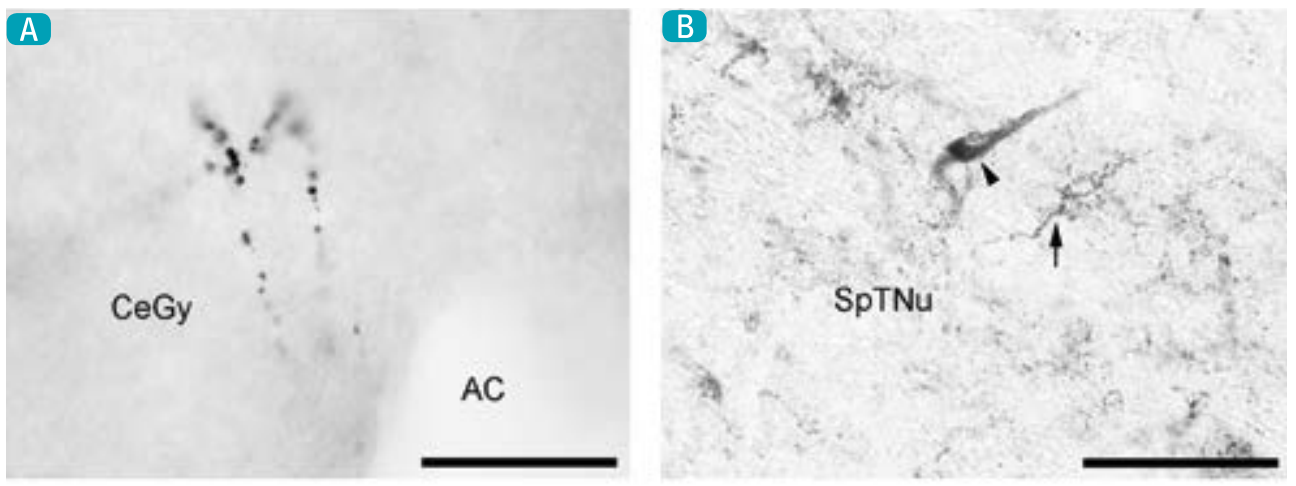

Figura I. Corte frontal del tronco del encéfalo humano. Las imágenes A y B muestran somas y fibras marcadas con $\alpha$-neo-endorfina. En A se observan fibras en la sustancia gris periacueductal (CeGy) y en B somas (cabeza de flecha) y fibras (flecha) marcadas en el núcleo espinal del trigémino (SpTNu). AC: acueducto cerebral. Escala de $50 \mu \mathrm{m}$.
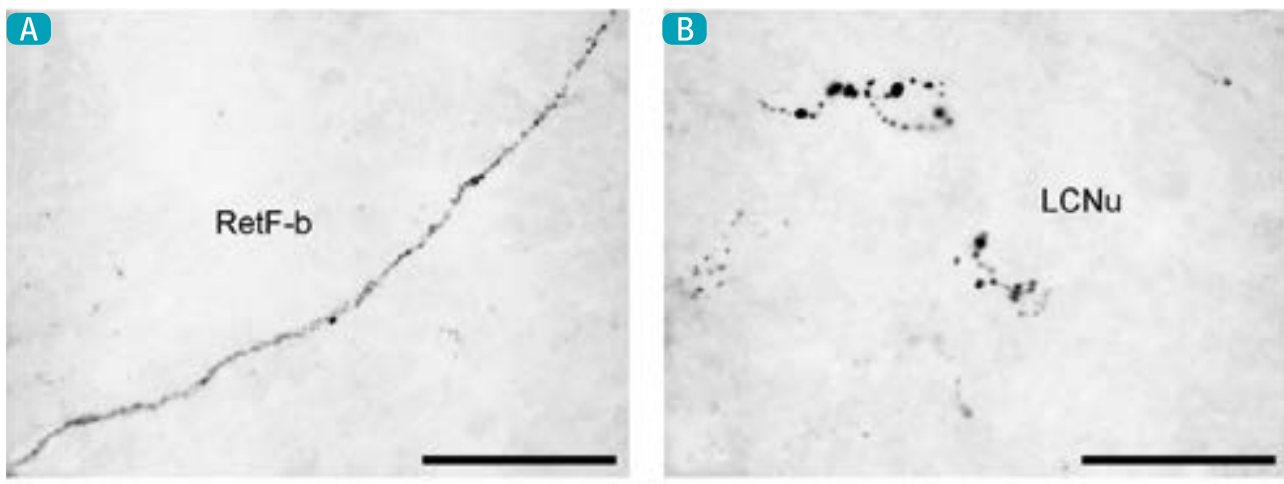

Figura 2. Corte frontal del tronco del encéfalo humano. Las imágenes A y B muestran fibras marcadas con $\alpha$-neoendorfina. En A se observan fibras en la formación reticular bulbar (RetF-b) y en B fibras marcadas en el núcleo cuneato lateral (LCNu). Escala de $50 \mu \mathrm{m}$.

Otra familia de neuropéptidos son las taquiquininas, entre las que se encuentran la sustancia $P$, la neuroquinina $A$, la neuroquinina $B$, la eledoisina, la bombesina y el péptido liberador de gastrina [3,16-19]. La sustancia $P$ es uno de los neuropéptidos más estudiados y fue uno de los primeros en aislarse; por ejemplo, Del Fiacco y colaboradores (1984) [16], usando técnicas de inmunocitoquímica, demostraron la presencia de estructuras inmunorreactivas (somas y fibras) marcadas con este neuropéptido en regiones del tronco del encéfalo humano como el colículo superior, la sustancia gris periacueductal, la formación reticular, el núcleo del tracto 
solitario, el núcleo espinal del trigémino y la sustancia negra. También se ha descrito la distribución de receptores para la sustancia $\mathrm{P}$ mediante experimentos de radioinmunoensayo en el tronco del encéfalo de niños que fallecieron por muerte súbita y en adultos [20-24]. En estos trabajos se demostró la presencia de receptores en núcleos como la sustancia gris periacueductal mesencéfalica, el locus coeruleus, el núcleo central superior, el núcleo hipogloso, el núcleo dorsal del rafe, el núcleo parabraquial, el núcleo del tracto solitario y el núcleo olivar inferior.

Duque y colaboradores (2013) [3], usando las técnicas de inmunocitoquímica, también describieron en el encéfalo humano de adultos post mortem la presencia de una baja densidad de somas y fibras marcados con neuroquinina B en la sustancia gris periacueductal, el colículo inferior, el núcleo ventromedial, el núcleo cuneato, el núcleo praepusitus, la formación reticular (mesencefálica, pontina y bulbar), la sustancia negra, el núcleo espinal del trigémino, el núcleo salivatorio inferior, el núcleo espinal vestibular inferior y el núcleo solitario (véase figura 3).
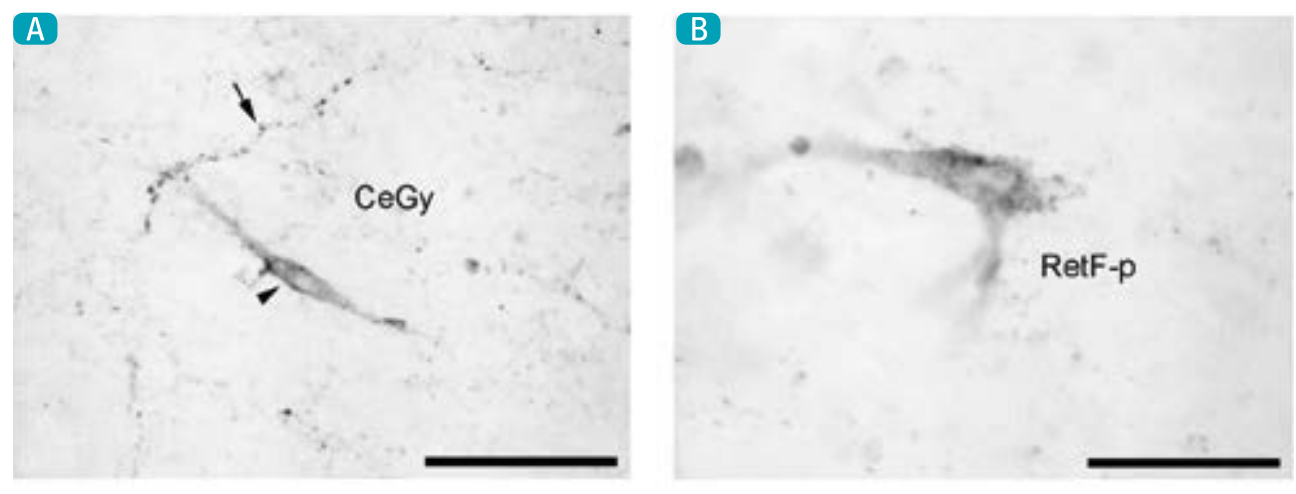

Figura 3. Corte frontal del tronco del encéfalo humano. Las imágenes A y B muestran somas y fibras marcadas con neurokinina B. En A se observan un soma (cabeza de flecha) y fibras (flecha) en la sustancia gris periacueductal (CeGy) y en B un soma marcado en la formación reticular pontina (RetF-p). Escala de $50 \mu \mathrm{m}$.

Existe otro neuropéptido entre los más estudiados, el neuropéptido Y, que es miembro de la familia del polipéptido pancreático, el cual está altamente concentrado en el hipotálamo. Por medio de radioinmunoensayos, Akabayashi y colaboradores (1994) [25] demostraron la presencia de receptores del neuropéptido $Y$ en el hipocampo (capas piramidales), la región de cabeza y cola del caudado, el locus coeruleus y la sustancia negra. Entretanto, utilizando la técnica de inmunocitoquímica e hibridación in situ se ha observado una amplia distribución del neuropéptido Y en los ganglios basales, la amígdala, el núcleo accumbens, el putamen, el giro dentado, el tálamo y las astas de amon, donde no sólo se han observado receptores a nivel de las astas de amon-I, sino somas y fibras inmunomarcadas en las astas de amon-I, 2 y 3 [26,27].

Además, mediante el uso de técnicas como el trac-tracing y la inmunocitoquímica, se han descrito circuitos y vías corticales que contienen el neuropéptido Y [28]. También, mediante hibridación in situ se encontraron altas concentraciones del neuropéptido $Y$ en la corteza frontal 
y parietal, el cuerpo amigdaloide y el giro dentado en el humano [29]. Otros estudios han evidenciado una alta densidad, así como una amplia distribución del neuropéptido Y en la formación hipocampal del humano adulto, especialmente en regiones como el complejo subicular y el complejo entorrinal, así como las astas de amón (subcapa astas de amon- I) y el haz angular $[30,31]$.

Otro neuropéptido es la somatostatina, un tetradecapéptido que fue originalmente aislado del hipotálamo ovino con base a su habilidad de inhibir la liberación de la hormona de crecimiento de las células de la hipófisis anterior [32]. Bouras y colaboradores (1987) [33] demostraron, por medio de inmunocitoquímica, la presencia del precursor de la somatostatina (prosomatostatina) en el sistema nervioso central del humano, encontrando somas marcados con prosomatostatina en áreas corticales y paleocorticales, la sustancia gris subcortical, el núcleo accumbens, el núcleo caudado, el putamen, el hipotálamo, el núcleo reticular talámico y la formación reticular. También, observaron fibras marcadas en regiones como los núcleos talámicos, el tronco del encéfalo y la médula espinal. Por su parte, por medio de un estudio autoradiográfico se ha descrito la presencia de receptores para la somatostatina en núcleos sensoriales, el complejo trigeminal, el núcleo del tracto solitario, el núcleo vestibular, el locus coeruleus, el complejo olivar inferior, la formación reticular, el núcleo parabraquial, entre otros $[34,35]$.

Por otro lado, Chigr y colaboradores (1989) [35] observaron estructuras inmunomarcadas con somatostatina en encéfalos de infantes, en los que se destacaba la presencia de este neuropéptido en regiones como la sustancia gris periacueductal, el núcleo praepusitus, el núcleo hipogloso, el núcleo cuneato, el núcleo del tracto solitario, el núcleo interpeduncular y el núcleo central superior. Además, otros autores observaron proyecciones de neuronas marcadas con somatostatina-28 en el prosencéfalo humano, describiendo somas y fibras ampliamente dispersos en el estriado ventral (núcleo accumbens, tubérculo olfatorio y base del núcleo de la estría terminal) [21,30].

Finalmente, se ha descrito la presencia en baja densidad de somas y fibras marcadas con neuropéptidos como la oxitocina [36], el factor liberador de corticotropina [36,37], la colecistoquinina [23,36] y la galanina [36], en diversas regiones del encéfalo como la sustancia gris periacueductal, el área tegmental ventral, el velo medular superior y el locus coeruleus.

En la figura 4 se muestra el porcentaje de núcleos con estructuras inmunorreactivas (somas y fibras) para los diferentes neuropéptidos. Así, se encuentra que el más alto porcentaje de núcleos marcados con somas corresponde a la somatostatina con un 23\%; mientras que el mayor porcentaje de núcleos marcados con fibras corresponde a la metionina-encefalina-8 con un $42 \%$. Estos altos porcentajes de núcleos en donde se observaron somas marcados con estos neuropéptidos sugieren que estas regiones pueden actuar como centros reguladores en la liberación de estas sustancias y que pueden proyectar axones hacia otras áreas del encéfalo.

En las tablas I y 2 se describe de manera resumida la distribución cartográfica de estructuras marcadas (somas y fibras) en el encéfalo humano, teniendo en cuenta el atlas estereotáxico de Haines [38]. 


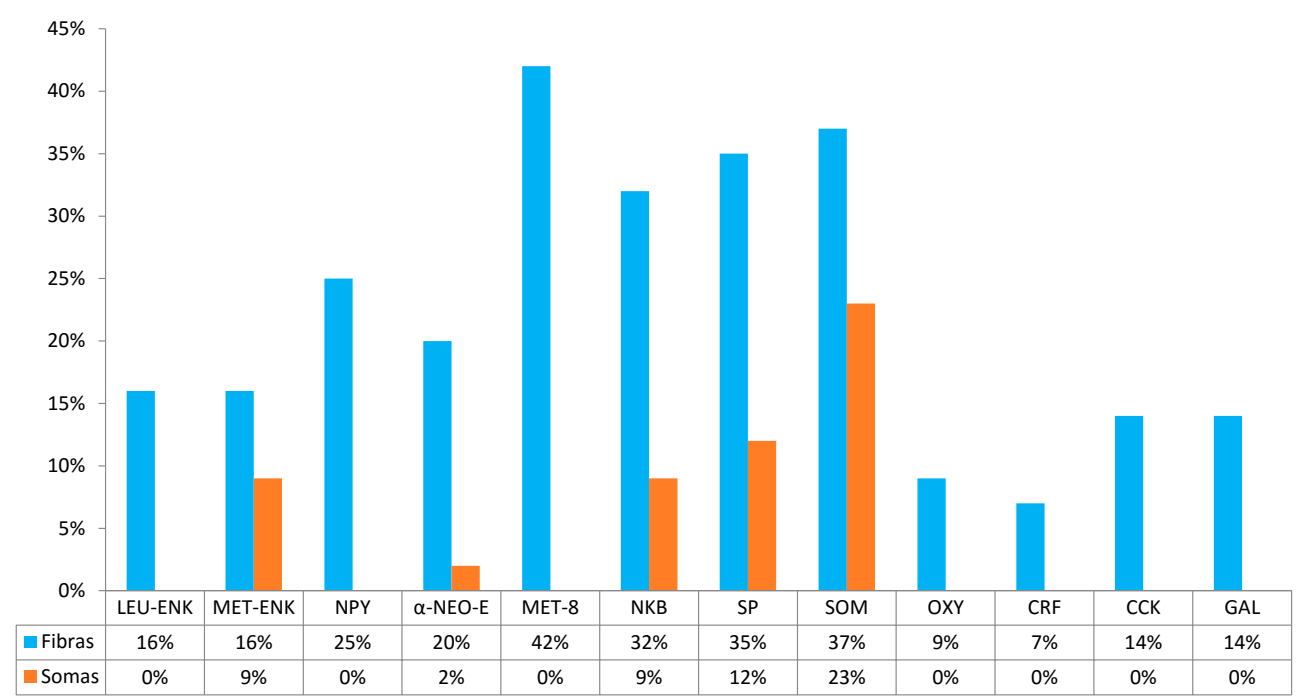

Figura 4. Porcentaje de estructuras inmunomarcadas (somas y fibras) con los diferentes neuropéptidos estudiados en el encéfalo humano. LEU-ENK: leucina-encefalina; MET-ENK: metionina-encefalina; NPY: neuropéptido Y; $\alpha$-NEO-E: a-neo-endorfina; MET-8: metionina-encefalina 8; NKB: neuroquinina B; SP: sustancia P; SOM: somatostatina; OXY: oxitocina; CRF: factor liberador de corticotropina; CCK: colecistoquinina; GAL: galanina.

\section{Tabla I. Distribución de neuropéptidos más comunes en los núcleos del encéfalo humano}

\begin{tabular}{|c|c|c|c|c|c|c|c|}
\hline \multirow{2}{*}{$\begin{array}{l}\text { Núcleos en el } \\
\text { encéfalo humano }\end{array}$} & $\begin{array}{l}\text { Leucina- } \\
\text { encefalina }\end{array}$ & $\begin{array}{l}\text { Metionina- } \\
\text { encefalina }\end{array}$ & L & the & $\begin{array}{l}\text { Metion } \\
\text { encefa }\end{array}$ & & \\
\hline & Fibras Soma & Fibras Soma & Fibras Soma & Fibras Soma & Fibras & Soma & \\
\hline
\end{tabular}

Astas de amon - $\quad$ - $\quad$ - $\quad$ - $\quad(R)$

R)

Globo pálido

(R)

Putamen

Núcleo caudado

R)

Ganglios basales

Núcleo amigdaloide

$(\mathrm{R})$

Núcleo dentado

Núcleo solitario

Núcleo hipogloso

(R)

(R)

Núcleo cuneato

Sustancia gris periacueductal

Núcleo prepósito

Núcleo del

tracto solitario

- $\quad(\mathrm{R})$

Núcleo espinal del trigémino

Formación reticular pontina

Formación reticular mesencefálica

Médula

Colículo superior

Colículo inferior

(R)

(R)

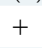

$+$

$-$

$+\quad+++$

$+$

(R)

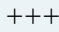

+++
$+++\quad-$
$+++\quad-$
$+++\quad-$

(R)
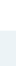

$++$

(R)

$++\quad+$

(R)

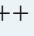

$++$

$+$

$++$

(R) 


\begin{tabular}{|c|c|c|c|c|c|c|c|c|c|c|c|c|}
\hline Sustancia negra & - & - & $(\mathrm{R})$ & - & (R) & - & - & - & +++ & - & ++ & - \\
\hline Locus coeruleus & - & - & $(\mathrm{R})$ & - & $(\mathrm{R})$ & - & - & - & - & - & - & - \\
\hline Oliva inferior & - & - & - & - & - & - & - & - & $+/+++$ & - & - & - \\
\hline Núcleo parabraquial & - & - & $(\mathrm{R})$ & - & - & - & - & - & - & - & - & - \\
\hline Núcleo cuneato lateral & - & - & $(\mathrm{R})$ & - & - & - & - & - & + & - & - & - \\
\hline Núcleo inrepeduncular & - & - & & - & - & - & - & - & $(\mathrm{R})$ & - & - & - \\
\hline Corteza & $(\mathrm{R})$ & - & $(R)$ & - & (R) & - & - & - & - & - & - & - \\
\hline Núcleo accumbens & + & - & - & - & (R) & - & - & - & - & - & - & - \\
\hline Núcleo vestibular & - & - & - & - & - & - & - & - & $+/+++$ & - & - & - \\
\hline Núcleo dorsal del rafe & - & - & - & - & - & - & - & - & +++ & - & - & - \\
\hline $\begin{array}{l}\text { Núcleo motor } \\
\text { dorsal del vago }\end{array}$ & - & - & - & - & - & - & + & - & - & - & - & - \\
\hline Núcleo gracili & - & - & - & - & - & - & + & - & - & - & - & - \\
\hline Núcleo ambiguo & - & - & - & - & - & - & + & - & - & - & - & - \\
\hline $\begin{array}{l}\text { Núcleo salivatorio } \\
\text { inferior }\end{array}$ & - & - & - & - & - & - & - & - & - & - & ++ & - \\
\hline $\begin{array}{l}\text { Núcleo vestibular } \\
\text { espinal inferior }\end{array}$ & - & - & - & - & - & - & - & - & - & - & ++ & - \\
\hline Hipocampo & - & - & - & - & (R) & - & - & - & - & - & - & - \\
\hline Tálamo & - & - & - & - & $(R)$ & - & - & - & - & - & - & - \\
\hline
\end{tabular}

\section{Tabla 2. Distribución de otros neuropéptidos en los núcleos del encéfalo humano}

\begin{tabular}{|c|c|c|c|c|c|c|c|c|c|c|c|c|}
\hline \multirow{2}{*}{$\begin{array}{l}\text { Núcleos en el } \\
\text { encéfalo humano }\end{array}$} & \multicolumn{2}{|c|}{ Sustancia P } & \multicolumn{4}{|c|}{ Somatostatina Oxitocina } & \multicolumn{2}{|c|}{$\begin{array}{l}\text { Factor liberador } \\
\text { de corticotropina }\end{array}$} & \multicolumn{4}{|c|}{ Colecistoquinina Galanina } \\
\hline & Fibras & Soma & Fibras & Soma & Fibras & Soma & Fibras & Soma & Fibras & Soma & Fibras & Soma \\
\hline Putamen & - & - & + & - & - & - & - & - & - & - & - & - \\
\hline Núcleo caudado & - & - & + & - & - & - & - & - & - & - & - & - \\
\hline Velo medular superior & - & - & - & - & + & - & $(\mathrm{R})$ & - & - & - & - & - \\
\hline Núcleo solitario & $(\mathrm{R})$ & - & $(\mathrm{R})$ & - & - & - & - & - & - & - & - & - \\
\hline Núcleo hipogloso & $(\mathrm{R})$ & - & + & - & - & - & - & - & - & - & - & - \\
\hline Núcleo cuneato & - & - & + & - & - & - & $(\mathrm{R})$ & - & $(\mathrm{R})$ & & $(\mathrm{R})$ & \\
\hline Sustancia gris periacueductal & + & + & + & - & + & - & - & - & + & & + & \\
\hline Núcleo del tracto solitario & + & - & + & - & - & - & - & - & - & - & - & - \\
\hline Núcleo espinal del trigémino & + & + & + & - & - & - & - & - & - & - & - & - \\
\hline Formación reticular pontina & + & - & - & + & - & - & - & - & - & - & - & - \\
\hline $\begin{array}{l}\text { Formación reticular mesen- } \\
\text { cefálica }\end{array}$ & + & - & - & + & - & - & - & - & - & - & - & - \\
\hline Médula & + & + & - & + & - & - & - & - & - & - & - & - \\
\hline Colículo superior & + & - & - & - & - & - & - & - & - & - & - & - \\
\hline Colículo inferior & $(\mathrm{R})$ & - & - & - & - & - & - & - & - & - & - & - \\
\hline Sustancia negra & - & - & $(\mathrm{R})$ & - & - & - & $(\mathrm{R})$ & - & $(\mathrm{R})$ & & $(\mathrm{R})$ & \\
\hline Locus coeruleus & $(\mathrm{R})$ & - & $(\mathrm{R})$ & - & + & - & - & - & - & - & - & - \\
\hline Oliva inferior & $(\mathrm{R})$ & - & $(\mathrm{R})$ & - & - & - & - & - & - & - & - & - \\
\hline Núcleo parabraquial & $(\mathrm{R})$ & - & $(\mathrm{R})$ & - & - & - & - & - & - & - & - & - \\
\hline Núcleo central superior & $(\mathrm{R})$ & - & - & - & - & - & - & - & - & - & - & - \\
\hline Núcleo inrepeduncular & - & - & + & - & - & - & - & - & - & - & - & - \\
\hline Núcleo reticular talámico & - & - & + & + & - & - & - & - & - & - & - & - \\
\hline
\end{tabular}


$(+)=$ presencia (+: baja densidad; ++: moderada densidad; +++: ata densidad)

$(-)=$ ausencia

(I) =proporción de la densidad entre soma o fibras encontrado

$(R)=$ presencia del receptor

\section{Posibles acciones fisiológicas de los neuropéptidos en el encéfalo humano}

La amplia distribución de los neuropéptidos en el encéfalo humano sugiere que algunas de estas sustancias están involucradas en un amplio número de funciones. Por ejemplo, en el humano la presencia de fibras que contienen metionina-encefalina-Arg-Gly-Leu ${ }^{8}$ encontradas en el núcleo espinal del trigémino sugiere una relación con los mecanismos de percepción del dolor y la temperatura. Además, la presencia de fibras marcadas con este neuropéptido en el núcleo solitario está relacionada con la sensibilidad visceral [13]. Se ha demostrado también que la presencia de somas y fibras marcadas con $\alpha$-neo-endorfina en varias regiones encefálicas están relacionadas con mecanismos nociceptivos (sustancia gris periacueductal, núcleo espinal del trigémino) y mecanismos visuales (colículo superior) [3].

Los niveles elevados de ARN mensajero de precursores neuropeptídicos como la prodinorfina han demostrado ser clave en los circuitos relacionados con el control de las emociones y la respuesta al estrés, por lo que se ha propuesto un rol importante de las dinorfinas en la ansiedad, el control emocional y los desórdenes sicóticos [39,40]. Además, se ha evidenciado el papel que desempeñan las taquiquininas como las neuroquininas en el sistema nervioso central. Es así como diferentes investigaciones han demostrado, por ejemplo, el papel de los receptores de neuroquinina-I unidos a la sustancia $P$, en la regulación de muchas acciones fisiológicas tales como el comportamiento emocional, la ansiedad, el vómito, la migraña, la adicción, las convulsiones y la neurodegeneración. Incluso, en algunos casos se ha observado que los eventos depresivos tienen como consecuencia la activación de la cascada sustancia P/neuroquinina-I, que se refleja en una inmunosupresión y un fenómeno apoptótico que pueda acarrear otro tipo de enfermedades como el cáncer, hepatopatías, infecciones, entre otros $[4 \mid]$.

Otros estudios han evidenciado que las taquiquininas se encuentran relacionadas con el eje reproductivo del sistema nervioso central $[42,43]$. Sin embargo, se demostró que la microinyección a nivel ventricular de neuroquina- I y neuroquinina $B$ produce un ascenso en la presión arterial y el ritmo cardíaco, que sugiere un posible papel de esta familia de neuropéptidos en la regulación de funciones autonómicas tales como los mecanismos de control cardiovascular y respiratorio [44]. Así mismo, se ha demostrado una bradipnea después de administrar mediante microinyección neuroquinina $B$ en el núcleo del tracto solitario [45]. De esta manera, y según los resultados de Duque y colaboradores (2013) [3], la presencia de fibras inmunomar- 
cadas con neuroquinina B en el núcleo del tracto solitario sugiere que este neuropéptido está relacionado con mecanismos respiratorios y cardiovasculares.

En el sistema nervioso central el neuropéptido Y es sintetizado en neuronas del núcleo arcuato, el cual proyecta a muchos otros núcleos hipotalámicos, incluyendo el núcleo paraventricular. El neuropéptido $Y$ exhibe bioactividad en múltiples sistemas en concentraciones nanomolares y altamente conservada a través de las especies, reflejando su posible importancia como un neuromodulador encefálico. Existe evidencia de la relación que tiene el neuropéptido Y con procesos fisiológicos como la ingesta de alimentos, el sistema endocrino y el ritmo circadiano [46]. Otros estudios han demostrado que la presencia de estructuras (fibras) inmunomarcadas con neuropéptido $Y$, en conjunto con el péptido intestinal vasoactivo y la sustancia $P$, alrededor de vasos sanguíneos que limitan con la corteza visual, pueden estar ejerciendo un papel en la regulación del flujo sanguíneo cortical [47]. Además, se ha demostrado la presencia de somas y fibras marcados con neuropéptido Y en los núcleos hipotalámicos, lo que sugiere un papel modulador de la acción neuroendocrina y otras funciones de regulación básicas concernientes a la ingesta de alimentos y líquidos [48,49].

La somatostatina desempeña un papel en el control inhibitorio de las hormonas hipotalámicas [50,5 I], aunque los resultados de otros estudios como la presencia de estructuras inmunomarcadas con somatostatina en el núcleo del tracto solitario del tronco del encéfalo de niños sugieren otras posibles acciones fisiológicas como el control respiratorio [35]. Finalmente, se sugiere que la somatostatina puede desempeñar un rol muy importante en la generación del sueño paradójico y la modulación de la integración central autonómica, los cual se soporta en estudios donde se ha observado, a través de autoradiografía, un alto número de receptores de somatostatina en el locus coeruleus [32,34].

\section{Coexistencia de neuropéptidos en el encéfalo humano}

La presencia de varios neuropéptidos en las mismas regiones y núcleos del encéfalo humano indican una posible interacción entre estos, así como una elaborada modulación en la cual están involucradas algunas regiones del encéfalo (véase tabla I). Además, la localización de varios neuropéptidos distintos en los mismos núcleos indica que dos a más de ellos pueden coexistir en la misma neurona. Por ejemplo, en el humano se ha encontrado la coexistencia de encefalinas (metionina-encefalina, leucina- encefalina y metionina-encefalina-8) y sustancia $P$ en el locus coeruleus y en el núcleo del tracto solitario $[8,10,11]$.

De acuerdo a la distribución y características peptidérgicas de los somas encontrados en el encéfalo del humano (véase tabla I), las siguientes son las posibles coexistencias que se pueden sugerir:

$\rightarrow \alpha$-neo-endorfina y neuroquinina B en la sustancia gris periacueductal [3].

$\rightarrow$ Sustancia P y somatostatina en el núcleo espinal del trigémino [ [6, 17,20,33,34].

$\rightarrow$ Neuroquinina B y somatostatina en la formación reticular pontina [3,34]. 
En orden a demostrar la coexistencia de los neuropéptidos indicados anteriormente, deben llevarse a cabo estudios a futuro usando doble marcaje inmunofluorescente.

\section{Investigaciones futuras sobre los neuropéptidos en el encéfalo humano}

Hay mucho por hacer a fin de obtener plena comprensión de la distribución y las funciones fisiológicas de los neuropéptidos en el sistema nervioso central del humano. Por lo tanto, además de estudiar la distribución de otros neuropéptidos, que en la actualidad no se han revisado en detalle, en el encéfalo humano, es importante a futuro desarrollar trabajos usando técnicas de radioinmunoensayo e hibridación in situ y poder relacionar los resultados obtenidos por estas técnicas con los descritos usando las técnicas de inmunocitoquímica.

Además, en el encéfalo humano deben ser explorados en profundidad aspectos que no han recibido suficiente atención como: a) la distribución de los receptores de los neuropéptidos, b) la distribución de las neuropeptidasas, c) la ubicación de los somas neuronales que originan aferencias peptidérgicas al encéfalo, d) las proyecciones de las neuronas peptidérgicas observadas en el encéfalo, e) las conexiones sinápticas que contienen neuropéptidos, f) la coexistencia de los neuropéptidos y g) las funciones fisiológicas de los neuropéptidos.

En resumen, se requiere la aplicación de otras metodologías que combinen los resultados de inmunocitoquímica y las vías de trazado retrógrado, así como métodos de microscopía electrónica, inmunofluorescencia, entre otros, a fin de obtener una mayor comprensión de la distribución y funciones de los neuropéptidos en el encéfalo humano.

\section{Conclusiones}

La amplia distribución de neuropéptidos en el encéfalo humano sugiere que estas sustancias neuroactivas podrían estar involucradas en varias acciones fisiológicas, actuando como neurotransmisores o neuromoduladores. Así, la presencia de neuropéptidos en regiones como el núcleo parabraquial sugiere que estos pueden estar mediando fenómenos respiratorios. Si bien se conoce la distribución de muchos neuropéptidos, aún hay mucho por explorar sobre este tema. El conocer más sobre la distribución de este tipo de sustancias en el sistema nervioso central permitirá establecer los mecanismos eficientes que puedan coadyuvar a la solución de problemas propios de la neurología como trastornos, neurodegeneración, entre otros.

\section{Agradecimientos}

Nuestros más sinceros agradecimientos a la Escuela de Medicina de la Universidad de Santander (UDES). Esta revisión ha sido apoyada por la Vicerrectoría de Investigaciones de la Universidad de Santander (UDES). 


\section{Bibliografía}

I. Coveñas R, Duque E, Mangas A, Marcos P, Narváez JA. Neuropeptides in the monkey (Macaca fascicularis) brainstem. En: Mangas A, Covenas R, Geffard M, eds. Brain Molecules: From Vitamins to Molecules for Axonal Guidance. India: Transworld Research Network; 2008: I31-156.

2. Duque E, Mangas A, Díaz-Cabiale Z, Narváez JA, Coveñas R. Neuropeptides in the Monkey Brainstem En: Williams RM, ed. Monkeys: Biology, Behavior and Disorders. Nueva York, Estados Unidos: Nova Science Publishers, Inc.; 20 I I: I 5 I - 166.

3. Duque E, Mangas A, Salinas P, Díaz-Cabiale Z, Narváez JA, Coveñas R. Mapping of alpha-neo-endorphin- and neurokinin B-immunoreactivity in the human brainstem. Brain Struct Funct 2013; 218: |3|-| 49 .

4. Coveñas R, Mangas A, Narváez JA. Introduction to neuropeptides. En: Coveñas R, Mangas A, Narváez JA, eds. Focus on Neuropeptide Research. India: Transworld Research Network; 2007: I-26.

5. Roumy M, Zajac JM. Neuropeptide FF, pain and analgesia. Eur J Pharmacol 1998; 345: I-I I.

6. Newton BW, Maley B, Sasek C, Traurig H. Distribution of FMRF-NH2-like immunoreactivity in rat and cat area postrema. Brain Res Bull 1984; I3: 39|-399.

7. Raffa RB. The action of FMRFamide (Phe-Met-ArgPhe-NH2) and related peptides on mammals. Peptides 1988; 9: 915-922.

8. Bouras $\mathrm{C}$, Taban $\mathrm{CH}$, Constantinidis J. Mapping of enkephalins in human brain. An immunohistofluorescence study on brains from patients with senile and presenile dementia. Neuroscience 1984; 12: 179-190.

9. Sapp E, Ge P, Aizawa H, Bird E, Penney J, Young $A B$, et al. Evidence for a preferential loss of enkephalin immunoreactivity in the external globus pallidus in low grade Huntington's disease using high resolution image analysis. Neuroscience 1995; 64: 397 404.

10. Rinne JO, Lonnberg P, Marjamaki P, Molsa P, Sako E, Paljarvi L. Brain methionine- and leucine-enkephalin receptors in patients with dementia. Neurosci Lett 1993; 161: 77-80.

II. Rinne JO, Lonnberg P, Marjamaki P. Human brain methionine- and leucine-enkephalins and their receptors during ageing. Brain Res 1993; 624: 131 136.

12. Iadarola MJ, Flores CM, Cadet JL, Suddath R, Illowsky B, Berman KF. Met5-enkephalin-Arg6Gly7-Leu8 immunoreactivity in rat and human cerebrospinal fluid: influence of neuroleptic drugs and electroconvulsive shock. Brain Res 1988; 474: 75-85.
13. Coveñas R, Martin F, Salinas P, Rivada E, Smith V Aguilar LA, et al. An immunocytochemical mapping of methionine-enkephalin-Arg(6)-Gly(7)-Leu(8) in the human brainstem. Neuroscience 2004; 128: 843-859.

14. Pasi A, Mehraein P, Jehle A, Traub M, Morniroli G, Bar W, et al. Beta-endorphin: regional levels profile in the brain of the human infant. Neurochem Int 1992; 20: 93-95.

15. Coquerel A, Buser M, Tayot J, Pfaff F, Matray F, Proust B. Beta-endorphin and neurotensin in brainstem and cerebrospinal fluid in the sudden infant death syndrome. Neurochem Int 1992; 20: 97-102.

16. Del Fiacco M, Dessi ML, Levanti MC. Topographical localization of substance $P$ in the human post-mortem brainstem. An immunohistochemical study in the newborn and adult tissue. Neuroscience 1984; |2: 59|-61|.

17. Chigr F, Najimi M, Leduque P, Chayvialle JA, Bouvier R, Kopp N. Anatomical distribution of substance P-immunoreactive neurons in human brainstem during the first postnatal year. Brain Res Bull 199|; 26: 515-523.

18. Muñoz M, Coveñas R. Involvement of substance $P$ and the NK-I receptor in human pathology. Amino Acids 20|4; 46: 1727-1750.

19. Marcos P, Coveñas R, de León M, Narváez JA, Tramu G, Aguirre JA, et al. Neurokinin A-like immunoreactivity in the cat brainstem. Neuropeptides 1993; 25: 105-I|4.

20. Del Fiacco M, Dessi ML, Atzori MG, Levanti MC. Substance $P$ in the human brainstem. Preliminary results of its immunohistochemical localization. Brain Res 1983; 264: 142-147.

2I. Biggins JA, McDermott JR, Smith Al, Candy JM, Perry RH, Bateman DE, et al. Chromatographic characterization of neuropeptides in post mortem human brain. J Neurol Sci 1984; 66: 47-58.

22. Bergstrom L, Lagercrantz H, Terenius L. Post-mortem analyses of neuropeptides in brains from sudden infant death victims. Brain Res 1984; 323: 279-285.

23. Kleinman JE, Hong J, ladarola M, Govoni S, Gillin C]. Neuropeptides in human brain--postmortem studies. Prog Neuropsychopharmacol Biol Psychiatry 1985; 9: 91-95.

24. Mai JK, Stephens PH, Hopf A, Cuello AC. Substance $P$ in the human brain. Neuroscience 1986; 17: 709-739.

25. Akabayashi A, Wahlestedt C, Alexander JT, Leibowitz SF. Specific inhibition of endogenous neuropeptide $Y$ synthesis in arcuate nucleus by antisense oligonucleotides suppresses feeding behavior and insulin secretion. Brain Res Mol Brain Res 1994; 21: 
$55-61$.

26. Yu MC, Luo CB, Long L, Yew DT. An immunohistochemical study of neuropeptide $Y$ positive sites in the developing human hippocampal formation. Brain Res Dev Brain Res 1993; 72: 277-281.

27. Caberlotto L, Fuxe K, Overstreet DH, Gerrard $P$, Hurd $Y L$. Alterations in neuropeptide $Y$ and $Y I$ receptor mRNA expression in brains from an animal model of depression: region specific adaptation after fluoxetine treatment. Brain Res Mol Brain Res 1998; 59: 58-65.

28. Haber S. Tracing intrinsic fiber connections in postmortem human brain with WGA-HRP. J Neurosci Methods 1988; 23: 15-22.

29. Brené S, Lindefors N, Kopp J, Sedvall G, Persson $\mathrm{H}$. Regional distribution of neuropeptide $Y$ mRNA in postmortem human brain. Brain Res Mol Brain Res 1989; 6: 241-249.

30. Gaspar P, Berger B, Lesur A, Borsotti JP, Febvret A. Somatostatin 28 and neuropeptide $Y$ innervation in the septal area and related cortical and subcortical structures of the human brain. Distribution, relationships and evidence for differential coexistence. Neuroscience 1987; 22: 49-73.

31. Lotstra F, Schiffmann SN, Vanderhaeghen JJ. Neuropeptide $\mathrm{Y}$-containing neurons in the human infant hippocampus. Brain Res 1989; 478: 21 I-226.

32. Carpentier V, Vaudry H, Mallet E, Laquerriere A, Tayot J, Leroux P. Anatomical distribution of somatostatin receptors in the brainstem of the human fetus. Neuroscience 1996; 73: 865-879.

33. Bouras C, Magistretti PJ, Morrison JH, Constantinidis J. An immunohistochemical study of pro-somatostatin-derived peptides in the human brain. Neuroscience 1987; 22: 781-800.

34. Carpentier V, Vaudry H, Laquerriere A, Tayot J, Leroux $\mathrm{P}$. Distribution of somatostatin receptors in the adult human brainstem. Brain Res 1996; 734: |35- 48.

35. Chigr F, Najimi M, Leduque $P$, Charnay $Y$, Jordan D, Chayvialle JA, et al. Anatomical distribution of somatostatin immunoreactivity in the infant brainstem. Neuroscience 1989; 29: 615-628.

36. Fodor M, Gorcs TJ, Palkovits M. Immunohistochemical study on the distribution of neuropeptides within the pontine tegmentum--particularly the parabrachial nuclei and the locus coeruleus of the human brain. Neuroscience 1992; 46: 89I-908.

37. Austin MC, Janosky JE, Murphy HA. Increased corticotropin-releasing hormone immunoreactivity in monoamine-containing pontine nuclei of depressed suicide men. Mol Psychiatry 2003; 8: 324-332.

38. Haines DE. Neuroanatomy: An Atlas of Structures, Sections, and Systems.
39. Schwarzer C. 30 years of dynorphins--new insights on their functions in neuropsychiatric diseases. Pharmacol Ther 2009; 123: 353-370.

40. Wittmann W, Schunk E, Rosskothen I, Gaburro $\mathrm{S}$, Singewald N, Herzog H, et al. Prodynorphin-derived peptides are critical modulators of anxiety and regulate neurochemistry and corticosterone. Neuropsychopharmacology 2009; 34: 775-785.

4I. Coveñas R, Martin F, Belda M, Smith V, Salinas P, Rivada $E$, et al. Mapping of neurokinin-like immunoreactivity in the human brainstem. BMC Neurosci 2003; 4: 3

42. Rance NE, Krajewski SJ, Smith MA, Cholanian M, Dacks PA. Neurokinin B and the hypothalamic regulation of reproduction. Brain Res 2010; 1364: I 16-128.

43. Topaloglu AK, Semple RK. Neurokinin B signalling in the human reproductive axis. Mol Cell Endocrinol 20II; 346: 57-64.

44. Picard P, Regoli D, Couture R. Cardiovascular and behavioural effects of centrally administered tachykinins in the rat: characterization of receptors with selective antagonists. $\mathrm{Br}$ J Pharmacol 1994; II2: 240-249.

45. Mazzone SB, Geraghty DP. Respiratory actions of tachykinins in the nucleus of the solitary tract: characterization of receptors using selective agonists and antagonists. Br J Pharmacol 2000; 129: I I21II3I.

46. Allen JM, Ferrier IN, Roberts GW, Cross AJ, Adrian TE, Crow TJ, et al. Elevation of neuropeptide $Y$ (NPY) in substantia innominata in Alzheimer's type dementia. J Neurol Sci 1984; 64: 325331.

47. Van Reeth O, Goldman S, Schiffmann S, Verstappen A, Pelletier G, Vaudry H, et al. Distribution of neuropeptide $Y$ immunoreactivity in human visual cortex and underlying white matter. Peptides 1987; 8: | |07-1 II7.

48. Walter A, Mai JK, Jimenez-Hartel W. Mapping of neuropeptide $Y$-like immunoreactivity in the human forebrain. Brain Res Bull 1990; 24: 297-3II.

49. Jacques D, Tong Y, Shen SH, Quirion R. Discrete distribution of the neuropeptide $Y$ Y5 receptor gene in the human brain: an in situ hybridization study. Brain Res Mol Brain Res 1998; 61: 100-107.

50. Shepherd GM. Neurobiology (ed 2a). Nueva York, Estados Unidos: Oxford University Press; 1988.

5I. Cebada-Sanchez S, Insausti R, Gonzalez-Fuentes J, Arroyo-Jimenez MM, Rivas-Infante E, Lagartos MJ, et al. Distribution of peptidergic populations in the human dentate gyrus (somatostatin [SOM28, SOM-12] and neuropeptide Y [NPY]) during postnatal development. Cell Tissue Res 2014; 358: 25-41. 
Laboratorio
Clinico
Hematológico

Creemos en los sueños de todos los colombianos que llevan en la sangre confianza

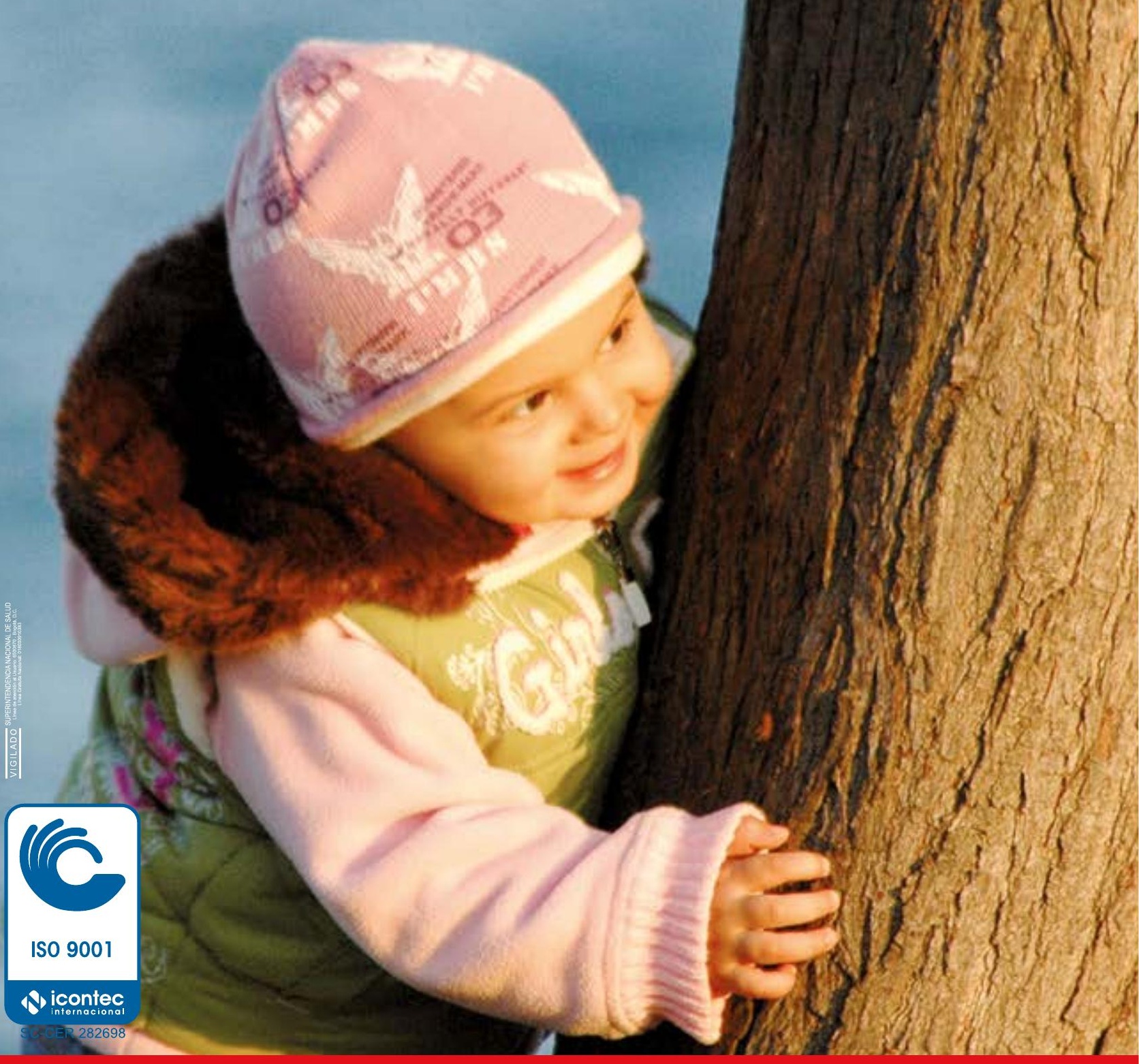

Sede Principal Poblado: Carrera 43C No. 5-33

Toma de Muestras: Centro Comercial Sandiego, Torre Norte, Piso 10, No. 1034 